\title{
AN X-RAY VIEW OF INTERACTING BINARIES OUTSIDE THE GALAXY
}

\author{
Andrea H. Prostwich ${ }^{1}$
}

RESUMEN

Chandra y XMM-Newton están alterando radicalnente nuestra mancra do cutender lat binarias compactas on galaxias externas, permitiéndonos estudiar detalladamente las fucnten con galaxias del (irupo Local. y tambiŕn las poblaciones en sistemas más lejanos. En M31 la función de luminosidad de ravos X dependede de la población

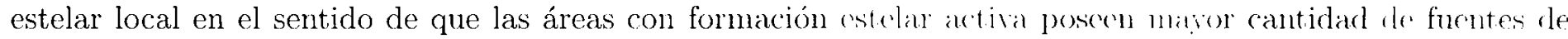
alta luminosidad y una mayor densidad total de fuontes (Kong. Di Stefino. CHarcía. A. Greiner 2003). Este resultado es también válido para galaxias fuera del Cirupo Local; las galaxiats con formación cistelar violonta

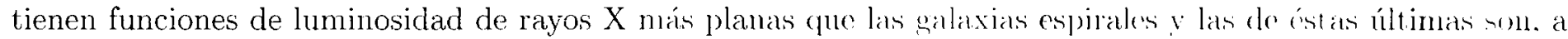

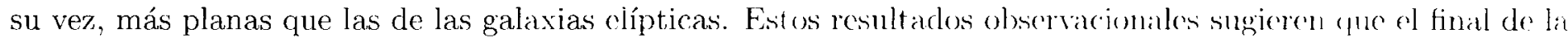
función de luminosidad de regiones de formación estelare está dominado por binarias de rayos $X$ de masas alt it y vidas de corta duración.

En el Ciclo 2 de Chandra hemos comenzado un extenso proverto para explorar una muestra de 11 galaxias espirales cercanas vistas de frente $(<10 \mathrm{Mpc})$. Encontranes que las fucutes so pueclen clasificar de manera aproximada en base a su color en rayos $\mathrm{X}$ en los grupos: binarias de rayos $X$ de miltat belja, binarias de rayess

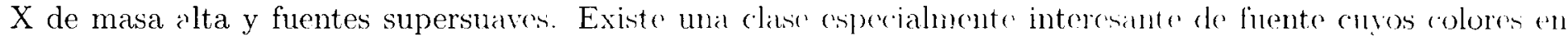
rayos $\mathrm{X}$ sor más suaves (más rojos) que los de una fuente típiea die rayos $X$ de baja miaria pero no llegann al extremo de las fuentes supersnaves. Isa mayoría son probablemente romanentes de supermovis brillantess en rayos $\mathrm{X}$, pero algunas pueden ser un nuevo tipo dr agujero wegro acrecicnte. Finalmente. comstruimns. una función de luminosidad seleccionando solamente aquellas fuentes con colores correspondienten al grupo de binarias de rayos $X$ de masa baja (quitando las fuentes suaves) encontrando que existe una caída o ruptura asociada probablemente con la luminosidad de Eddington de una cstrella de neutrones.

\section{ABSTRAC'T}

Chandra and XMIN-Newton are revolutionizing our understanding of compact binarien in external galaxies. allowing us to study sources in detail in Local Group Galaxies and st udy populations in more distiun systems. In M31 the X-ray luminosity function depends on the local stellar population in the sense thal arras with active star formation have more high luminosity souress, and a higher overall source density (Kong. Di Stefano. Garcia, \& Greiner 2003). This result is also true in galaxies outside the Local Group: starburst walexies hiure flatter X-ray luminosity functions than do spiral galaxies which are in turn flatter than elliptical galitxien. The'se observational results suggest that the high end of the luminosity function in star forming rewions is dominated by short-lived high mass X-ray binaries.

In Chandra Cycle 2 we started a Large Project to survey a sample of 11 marly (< 10.4 pe) fince-on -piral galaxies. We find that sources can be approximately classificd on the hasis of their X-ray color into low masis X-ray binaries, high mass X-ray binaries and supersoft sources. There is an especially interesting clitsis of source that has X-ray colors softer ("redder") than a typical low mass X-ray binary source. but not so extreme as supersoft sources. Most of these are probably X-ray bright supernova romants. but some may be' a new type of black hole accretor. Finally, when we construct a luminosity function of sourers selerting only sonrces with low mass X-ray binary colors (removing soft somres) we find that there is a dip or break probably unswiated with the Eddington luminosity for a neutron stitr.

\section{Key Words: X-RAYS: BINARIES}

\section{INTRODUCTION}

Galaxies contain a multitude of X-ray sources. In the Milky Way and MI31 the brightest of these: sources are Low Mass X-ray Binaries (L.IXB).

\footnotetext{
${ }^{1}$ Harvard-Smithsonian Center for Istrophysirs
}

accortion sistems where material is trantented ria Roche Lobe werflow from a low mass atar onte a compact companion (a white dwart, netutron star or black bole). High Iilss X-ray Binary (HMAXB) someress. systems powered be Bondi-Horle accretion 
from the stellar winds of a young, high mass star onto a compact object, are seen in star forming regions. Supernova remnants (SNR) are also known to be strong $\mathrm{X}$-ray sources. In this review, I focus studies of binary populations in M31 and in galaxies beyond the Local Group. A recent and very comprehensive overview of this subject can be found in Fabbiano \& White (2003).

\section{THE X-RAY BINARY LUMINOSITY FUNCTION IN M31 AND BEYOND}

M31 is the nearest spiral galaxy to our own, and is similar in size and metallicity to the Milky Way. However, it is possible to observe X-ray sources across the whole galaxy unlike in the Milky Way where obscuration in the galactic plane hides a large fraction of the binary population. It is therefore a key object for population studies, and has been observed extensively with both Chandra and XMMNewton (e.g. Garcia et al. 2000, Trudolyubov et al. 2002, Kong et al. 2002)

One of the most interesting results to emerge from recent studies is that the $\mathrm{X}$-ray luminosity function (XIF) depends on the local stellar population. Kong, Di Stefano, Garcia, \& Greiner (2003) surveyed three fields in the disk of M31. Field 1 was in the SW portion of the disk, and has very little evidence for star formation. Field 2 has many OB associations and optical SNR, suggestive of a young stellar population. Field 3 has a mixture of old and young stellar populations. The slopes of the XLFs in the three areas are $\alpha=1.7$ for field $1, \alpha=1.1$ for field 3 , and $\alpha=0.9$ for field 2 . Hence the area with the most active star formation (field 2) has the flattest luminosity function. In addition, field 2 has the highest density of X-ray sources. The region with the most active star formation has relatively more high luminosity sources, and a higher overall source density. This strongly suggests that the X-ray source population is closely tied to recent star formation.

This effect (flatter XLF in regions of star formation) can be understood qualitatively with simple analytical models of binary formation in which $\mathrm{X}$-ray sources are formed with a simple power law distribution in luminosity, and the highest luminosity sources die first (Kilgard et al 2002, Wu 2001, Schwartz et al 2003). The exact shape of the luminosity function depends on whether the star formation is constant or occurs in bursts. but in general as the population ages the luminosity function will steepen, and there will be a break in the LF corresponding to the last major episode of star formation. These simple models are illustrative, but very limited. Detailed population synthesis models are currently being prepared by Kalogara, Belczynski, Rasio and collaborators (see review by Kalogera in this volume and Belczynski, Kalogera, Zezas, \& Fabbiano 2003).

The XLF is intimately related to star formation in galaxies beyond the local group, as well as in M31. In a survey of nearby galaxies Kilgard et al (2002) found that the XLF of starburst galaxies was flatter than the XLF of spirals, which in turn are flatter than ellipticals, In addition, they found that the XLF is correlated with the far infrared luminosity, a good indicator of star formation activity. Colbert et al (2003) found that the combined X-ray luminosity of point sources $\left(L_{X P}\right)$ in star forming galaxies is correlated with the UV and FIR luminosity. These observational results suggest that the high end of the XLF in star forming galaxies is dominated by HMXBs. A comparison of the XLF of HMXBs in the milky way and more extreme star forming galaxies has led to the suggestion that there is a universal HMXB luminosity function. If this is true, $L_{X P}$ could be used as a star formation indicator (Grimm et al 2003.)

\section{A CHANDRA SURVEY OF NEARBY SPIRAL GALAXIES}

In Chandra Cycle 2 we started a Large Project to survey a sample of 11 nearby $(<10 \mathrm{Mpc})$ face-on spiral galaxies. Our sample spans the Hubble Sequence, and hence a range of star formation rates. Our limiting flux is $\sim 6 \times 10^{36} \mathrm{ergs} \mathrm{s}^{-1}$, which resolves approximately $80 \%$ of the discrete source flux. We have identified 822 unique souces within the galaxian $D_{25}$ ellipses (Kilgard et al 2004). One of the main objectives of the survey is to classify X-ray sources into broad categories, including LMXB, HMXB, SNR, supersoft sources and absorbed sources. We can then hope to understand how the individual populations depend on the current star formation rate and star formation history.

\subsection{Source Classification from $X$-ray Colors}

Figure 1 shows the X-ray color-color diagram for sources from a sample of spiral galaxies (see Prestwich et al 2003 for color definitions). The black crosses show sources from bulge systems and the red crosses show sources from disk systems. There is a statistically significant difference between the two distributions. The bulge sources cluster in a region with X-ray soft color between -0.4 to 0.2 , with a few very soft sources (at $0,-1)$. The disks, however, have an additional population of sources with soft color $=-0.9$ to -0.4 , not seen in bulges. See 


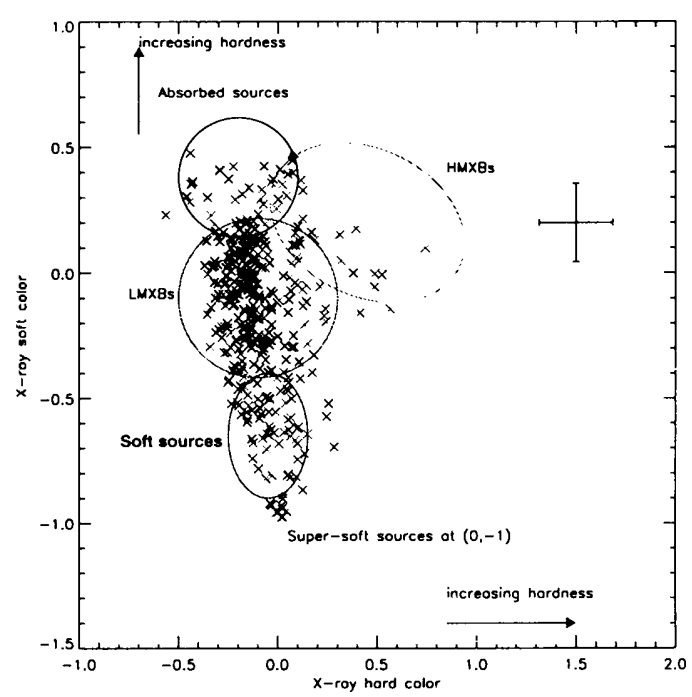

Fig. 1. X-ray color-color diagram.

section 3.2 for a more detailed discussion of these sources soft or "quasi-soft" (Di Stefano, Friedman, Kundu, \& Kong 2003) sources. Disks also have sources with X-ray hard color $\gtrsim 0.1$, not seen in bulges. These differences provide the basis for a source classification scheme. It is well known from observations of the Milky Way and Local Group galaxies that spiral bulges are dominated by LMXB sources, therefore it is plausible that many/most of the sources with soft color between -0.4 and 0.2 are LMXBs. The hard sources (X-ray hard color $\gtrsim 0.1$ ) seen in disks are logically identified with HMXBs. HMXBs have hard spectra (photon index $\Gamma \sim 1.0$ plus absorption) with X-ray colors that are consistent with those of the "hard" disk sources. In addition, we might expect to find HMXBs in regions of star formation since they have short-lived high mass secondaries. Finally, the softest sources (those with colors $0,-1$ ) are probably classical supersoft sources. These sources have most of their flux below $1 \mathrm{keV}$, and are highly variable. They are thought to be binaries with white dwarf primaries accreting at a highly super Eddington rate (Greiner et al 1991).

\subsection{The Nature of Soft Sources}

The nature of the "soft" or "quasi-soft" sources - those with soft X-ray color between -0.9 and -0.4 - are especially interesting. They are found in spiral bulges and disks, but appear to be more common in disks (Prestwich et al 2003). In the disks they follow the spiral arms which suggest that they are associated with the young stellar population. A few are clearly variable. A preliminary study by Kilgard et al (2004) suggests that at least $15 / 140$ soft sources in our sample are variable at the $90 \%$ level, compared to $112 / 450$ sources that are in the "LMXB" region of the color-color diagram, suggesting that as a class soft sources are less variable than LMXBs. However, at the time of writing we do not have good constraints on the variability of many soft sources, so this result needs to be confirmed. The soft sources in our sample typically have luminosities in the range $10^{36}-10^{37}$ ergs s$^{-1}$, and there may be many more below our detection limit. There are few soft sources with X-ray luminosities $\gtrsim 10^{37} \mathrm{ergs} \mathrm{s}^{-1}$, again in contrast to LMXB sources which have many sources in this range.

Sources that are classified as "soft" are undoubtedly made up of more than one type of object. For example, those that are variable are almost certainly accretion sources. Some of the variable soft sources may be classical supersoft sources viewed through material which preferentially absorbs the lowest energy photons making the X-ray colors somewhat less extreme. However, the classical model for supersoft sources will not work for most of the disk sources these are likely to be too young for a white dwarf to have formed, requiring a black hole or neutron star secondary (Di Stefano \& Kong 2003). Some may have an intrinsically soft, but not supersoft, spectrum. One particularly exciting possibility is that some of these sources represent a new class of black hole accretors, not previously identified in the Milky Way. A less exciting scenario is that the soft sources in disks are dominated by X-ray SNR. X-ray bright SNR have soft spectra, luminosities in the range $10^{36}-10^{37}$ ergs s$^{-1}$, and (assuming that they are old enough to be expanding adiabatically) are unlikely to exhibit variability. In addition, we would expect to see them in regions of current star formation, such as the spiral arms. Kilgard et al (2004) determined that the X-ray spectra of the brightest sources are best fit by a model which includes a MEKAL component, characteristic of optically thin line emission from hot gas. All these points strongly suggest that SNR are an important contributor to the class of soft sources. More detailed variability studies are needed to determine which (if any) type of object dominates the class of soft sources.

\subsection{Color Segregated Luminosity Functions - Eddington break?}

While the nature of the soft sources is uncertain, it is clear that most of them are not LNXBs. It is therefore instructive to construct a luminosity function with only with sources that have "LMIXB" colors, in the hope that by eliminating soft sources we select a cleaner sample of LMXBs. The color segregated LF for M83 is shown in Figure 2. The top 


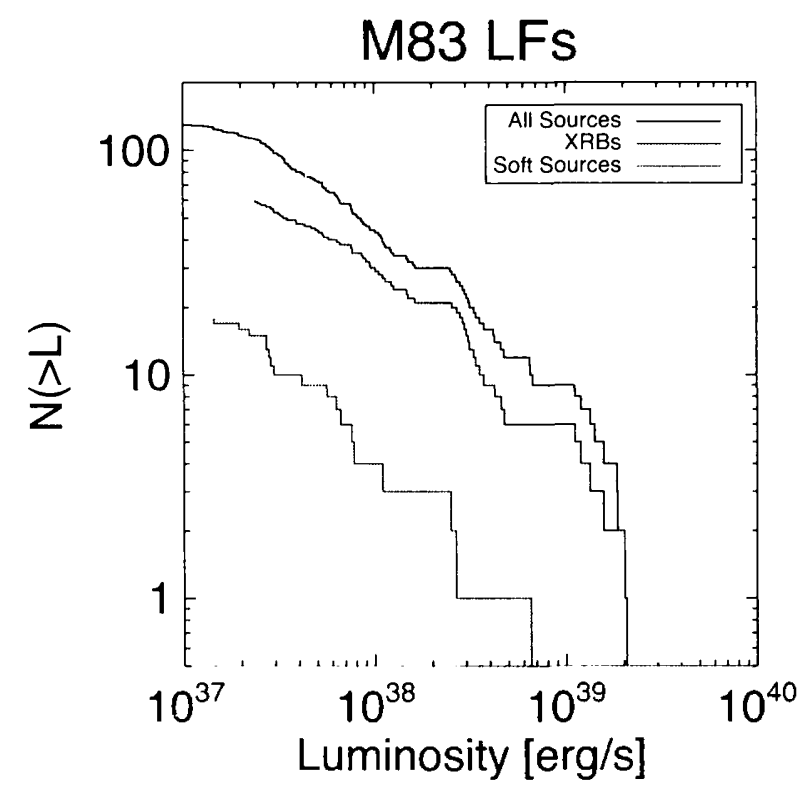

Fig. 2. Color segregated luminosity functions for M83.

curve shows all the sources in M83, the bottom curve just the soft sources and the middle curve sources with X-ray colors which put them in the "LMXB" part of the X-ray color-color diagram. With the soft sources removed, there appears to be a dip in the luminosity function of LMXB sources around $4 \times 10^{38}$ ergs $s^{-1}$. As discussed in Section 2, this feature is most likely to be either due to a aging population of binaries or a break at the Eddington luminosity for a neutron star. To distinguish between the possibilities, we constructed a luminosity function coadding all X-ray sources from our sample of galaxies which have "LMXB" color's. A preliminary plot is shown in Figure 3. Here the dip is clearly seen, suggesting that it is issociated with the Eddington break. This break has been observed in elliptical galaxies (e.g. Kim \& Fabbiano 2003, Sarazin, Irwin \& Bregman 2001) but not in spirals. It raise's the exciting possibility that at least some sources above the break are black hole accretors.

Thanks to Roy Kilgard, Rosamne Di Stefano, Hans-Jakob Grimm, Ben Williams and Albert Kong for useful discussions. This work wass supported by NASA contract NAS 8-39073 (CXC) and GO12029A.

\section{REFERENCES}

Belczynski, K., Kalogera, V., Z(æas. A., \& Fabbiano, G. 2003, ArXiv Astrophysics e-prints, astro-ph/0310200

Colbert, E., Heckman, T., Ptak, A.. Strickland, D., \& Weaver, K. 2003, ArXiv Astrophysics e-prints, astro$\mathrm{ph} / 0305476$

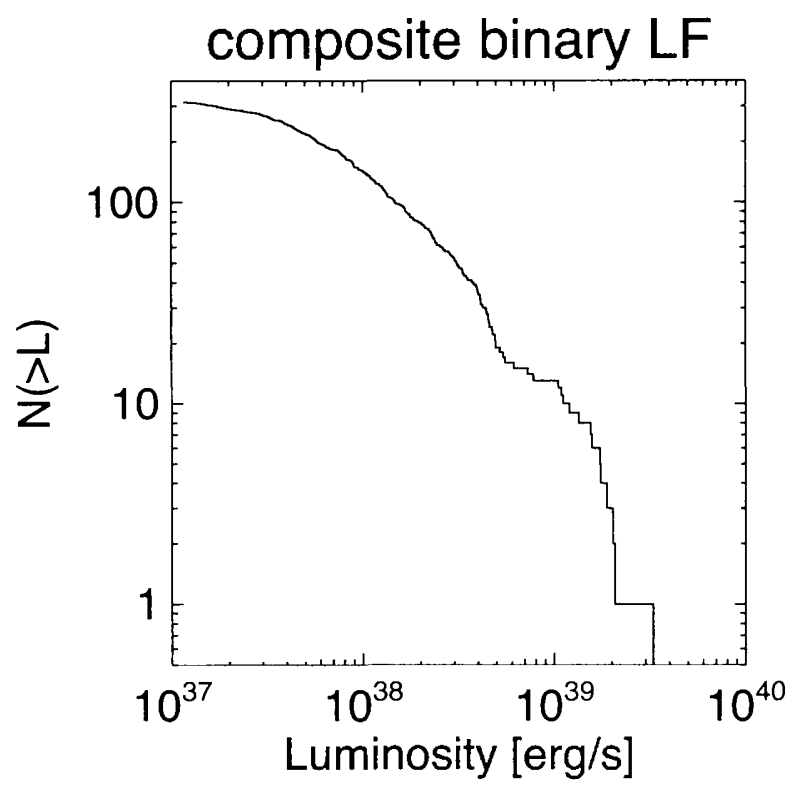

Fig. 3. Composite LMXB luminosity function.

Di Stefano, R., Friedman, R., Kundu, A., \& Kong, A. K. H. 2003, ArXiv Astrophysics e-prints, astroph/0312391

Di Stefano, R. \& Kong, A. K. H. 2003, ApJ, 592, 884

Fabbiano, G. \&. White, N. E. 2003, ArXiv Astrophysics e-prints, astro-ph/0307077

Garcia, M. R., Murray, S. S., Primini, F. A., Forman, W. R., McClintock, J. E., \& Jones, C. 2000, ApJ, $537, \mathrm{~L} 23$

Greiner, J., Hasinger, G., \& Kahabka, P. 1991, A\&A, 246, L17.

Grimm, H.-J., Gilfanov, M., \& Sunyaev, R. 2003, MNRAS, 339, 793

Kim, D. \& Fabbiano, G. 2003, ArXiv Astrophysics eprints, astro-ph/0312104

Kilgard, R. E., Kaaret, P., Krauss, M. I., Prestwich, A. H., Raley, M. T., \& Zezas, A. 2002, ApJ, 573, 138

Kilgard, R. E, Prestwich, A. H., Krauss, M. I., Ward, M., Zezas, A. et al submitted for publication in ApJ.

Kong, A. K. H., Garcia, M. R., Primini, F. A., Murray, S. S., Di Stefano, R., \& McClintock, J. E. 2002, ApJ, 577, 738

Kong, A. K. H., DiStefano, R., Garcia, M. R., \& Greiner, J. 2003, ApJ, 585, 298

Prestwich, A. H., Irwin, J. A., Kilgard, R. E., Krauss, M. I., Zezas, A., Primini, F., Kaaret, P., \& Boroson, B. 2003, ApJ, 595, 719

Sarazin, C. L., Irwin, J. A., \& Bregman, J. N. 2001, ApJ, 556,533

Swartz, D. A., Ghosh, K. K., McCollough, M. L., Pannuti, T. G., Tennant, A. F., \& Wu, K. 2003, ApJS, 114. 213

'Trudolyubov, S. P., Borozdin, K. N., Priedhorsky, W. C., Mason, K. O., \& Cordova, F. A. 2002, ApJ, 571, L17

Wu, K. 2001, Publications of the Astronomical Society of Australia, 18, 443 